\title{
Catalysing Mirror Life
}

\author{
Marleen Renders ${ }^{[c]}$ and Vitor B. Pinheiro ${ }^{*[a, b]}$
}

Information storage and propagation are central to life. The central dogma ${ }^{[1]}$ summarises the information flow of current biology, with two of the three information biopolymers (DNA, RNA and proteins) capable of propagation. The result is our traditional view of biology with DNA, the key repository of genetic information (genotype), going to RNA, going to proteins, the main source of functional molecules (phenotype).

The current information flow topology is complex and highly unlikely to have been the first to emerge on Earth. A simpler topology is contained within the central dogma as RNA can propagate (providing a genotype) and catalyse chemical reactions (providing a phenotype)—-the basis for the "RNA world" hypothesis. ${ }^{[2]}$

The "RNA world" hypothesis provides a sound framework for how life could have systematically evolved to our current biology. However, it highlights that little is known about how a biological system can emerge from mixtures of potential chemical building blocks ${ }^{[3]}$-including explaining the homochirality of current biopolymers (with $\mathrm{D}$ nucleic acids and L amino acids).

Synthetic routes to RNA nucleosides that are compatible with early Earth ${ }^{[3,4]}$ make use of achiral starting products and would be expected to achieve racemic mixtures of $D$ and $L$ precursors unless small enantiomeric biases could be amplified. ${ }^{\left[{ }^{[5]}\right.}$

Sczepanski and Joyce ${ }^{[6]}$ propose an alternative route. Although enantiomeric mixtures of activated precursors can interfere with the nonenzymatic, template-dependent polymerisation of nucleic acids, ${ }^{[7]}$ Sczepanski and Joyce demonstrate that a cross-chiral system would be viable-in which a D-RNA enzyme catalyses the replication of an L-RNA enzyme, which itself can catalyse the D-RNA enzyme assembly.

To achieve this elegant result, the authors initially used a strategy analogous to the selection of spiegelmers ${ }^{[8]}-\mathrm{L}-\mathrm{RNA}$ aptamers obtained through the selection of D-RNA aptamers against a chiral version of the desired target. Effectively, selection of a D-RNA nucleic acid enzyme (NAzyme) capable of ligating L-RNA also identifies an L-RNA NAzyme capable of DRNA ligation.

Dr. V. B. Pinheiro

Structural and Molecular Biology Department, University College London Gower Street, London, WC1E 6BT (UK)

E-mail:v.pinheiro@ucl.ac.uk

[b] Dr. V. B. Pinheiro

Institute of Structural and Molecular Biology

Department of Biological Sciences, Birkbeck College, University of London Malet Street, London, WC1E 7HX (UK)

[c] Dr. M. Renders

Laboratory for Medicinal Chemistry, Rega Institute for Medical Research Minderbroedersstraat 10, 3000, Leuven (Belgium)
Starting from a large $\mathrm{N}_{70}$ library, Sczepanski and Joyce selected for D-RNA NAzymes capable of ligating a 5'-triphosphorylated L-RNA biotinylated donor to an L-RNA acceptor hairpin (forming a double-stranded hairpin L-RNA) that itself is tethered via a linker to the D-RNA library (Figure 1).

Recovery of the biotinylated donor enabled partitioning of the NAzyme library and enrichment for active ligases. After six rounds of purifying selection, error-prone PCR was used to rediversify the library and explore the sequence neighbourhood of the partially selected repertoires. A further four rounds of selection with increased stringency (shortening the ligation reaction time from $16 \mathrm{~h}$ up to round 6 , to two hours in round 7 , and five minutes in rounds 8-10) were carried out before the selected repertoire was cloned and sequenced.

A catalytic motif was identified and used as the starting point for further engineering. The motif was partially optimised-some extraneous residues were removed-and further diversity $\left(\mathrm{N}_{30}\right)$ was added-as an insertion at a region expected to be single stranded and close to the NAzyme catalytic site. Six further rounds of directed evolution were carried out with increasing selection stringency and the introduction of repertoire diversity by error-prone PCR. Nonessential residues were removed to give a characterised 83-mer ribozyme-labelled 16.12t - capable of ligating L-RNA molecules through a $5^{\prime}-3^{\prime}$ linkage. The catalytic rate for the intramolecular reaction (RNAzyme tethered to one of the L-RNA primers) was determined to be $0.45 \mathrm{~min}^{-1}$ in a solution containing $250 \mathrm{mM} \mathrm{Mg}^{2+}$; this is a vast improvement $\left(10^{6}\right.$-fold) over the uncatalysed reaction. The catalytic rate for the intermolecular reaction is $0.019 \mathrm{~min}^{-1}$, which is comparable to previously isolated RNA ligase ribozymes. $^{\text {[9] }}$

Solid-phase synthesis was used to isolate the mirror-image NAzyme, an L-RNA enzyme capable of D-RNA ligation, enabling cross-catalysis and catalytic cycles to be investigated.

Although selected as a ligase, the $16.12 \mathrm{t}$ ribozyme was also capable of template-dependent polymerisation of L-RNA triphosphates starting from an L-RNA primer-template complex, even in the presence of D-RNA nucleotides. Incorporation rates vary significantly between the different triphosphates, with LCTP and L-GTP being the best-this can be explained if selection, always as a $\mathrm{C}-\mathrm{G}$ link, introduced a systematic bias.

Nevertheless, the flexibility of the enzyme-to be able to catalyse ligation of monomers as well as oligomers-enabled Sczepanski and Joyce to demonstrate a full replication cycle with the D-RNA NAzyme catalysing the synthesis of an active L-RNA ligase from a combination of activated monomers and oligomers.

The absence of cross-chiral inhibition allows the suggestion that both $\mathrm{D}$ - and L-RNA enantiomers might have been present 


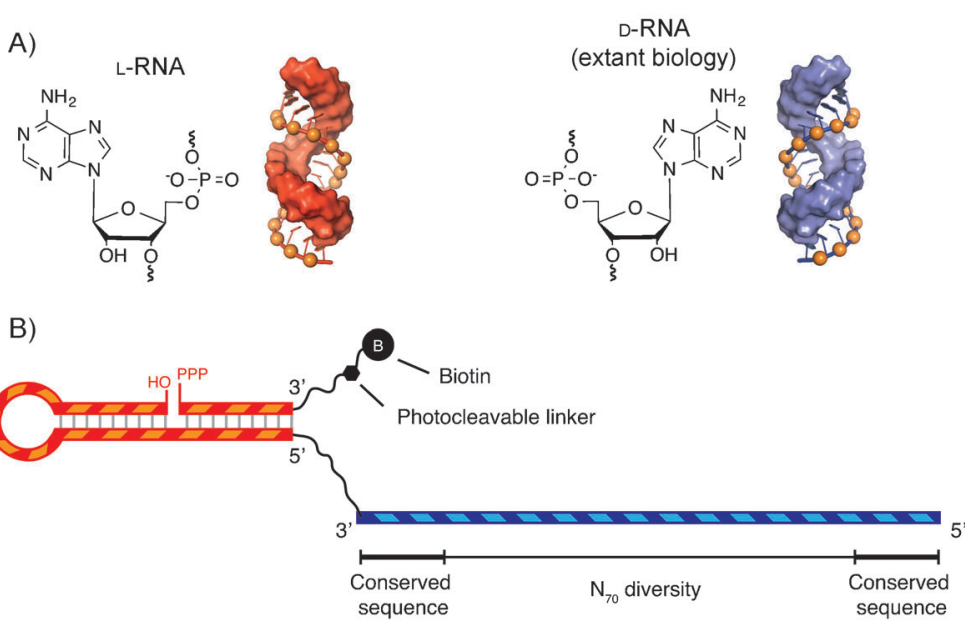

C)
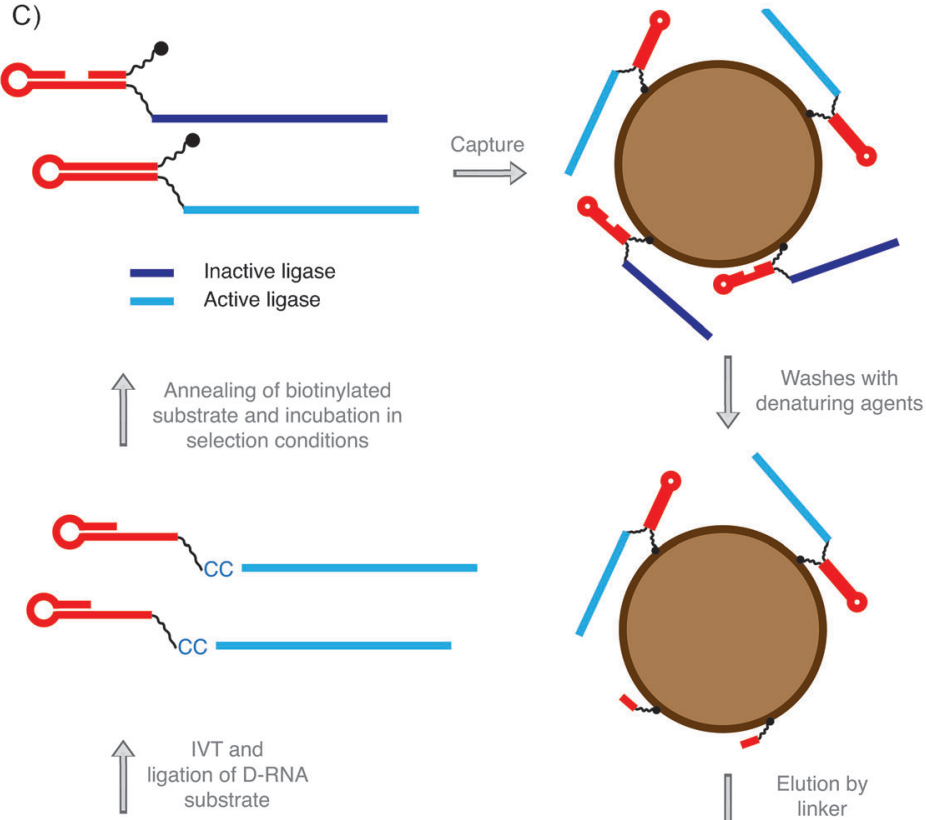

either in a pre-RNA world run by an achiral genetic material, or under conditions under which crosschiral precursor inhibition is not relevant or can be circumvented.

For instance, the cross-chiral inhibition reported in nonenzymatic, template-dependent nucleic acid synthesis ${ }^{[7]}$ used a methylimidazolide to activate a $5^{\prime}$ phosphate group in the nucleoside precursor, analogous to the pyrophosphate leaving group used by current biology to replicate nucleic acids. In that arrangement, cross-chiral precursors act as chain terminators, inhibiting replication. However, current scenarios suggest that a $3^{\prime}-2^{\prime}$ cyclic phosphate could have been a different route towards activating precursors, ${ }^{[10]}$ one that would not be expected to lead to cross-chiral inhibition.

As Sczepanski and Joyce highlight, a cross-chiral ribozyme ligase is less susceptible to sequence biases given that no two consecutive Watson-Crick base pairs are possible between a D- and an L-RNA molecule. That suggests possible routes to solve a different problem: how to further improve current ribozyme D-RNA polymerases. ${ }^{[11]}$ Synthetic genetic materials (XNAs) ${ }^{[12]}$ that do not form Watson-Crick base pairs with natural DNA or RNA molecules could be introduced in selection, substituting the L-RNA hairpin used by Sczepanski and Joyce.

Artificial base pairs that do not disrupt nucleic acid structure but alter major- and minor-groove contacts, such as the artificially expanded and the self-avoiding base pairs developed by Benner and colleagues, ${ }^{[13]}$ could be introduced in selection to stop consecutive Watson-Crick base pairing and thus minimise evolutionary biases that favour direct interaction with the template and primer.

Importantly, cross-chiral ligases are also the gateway towards establishing a mirror-world-biology running on $\mathrm{L}$ nucleic acids and $\mathrm{D}$ amino acids, unable to interact with our biology but where all our current knowledge of biological processes is readily applicable.

\section{Acknowledgements}

M.R. is supported by a post-doctoral fellowship from the FWO-Research Foundation Flanders (1247114N). V.B.P. acknowledges the ERC (StG 336936-HNAepisome) and $B B S R C(B B / K 018132 / 1)$ for their support.

Keywords: directed evolution - origin of life ribozyme $\cdot$ RNA world

[1] F. Crick, Nature 1970, 227, 561-563.

[2] a) R. F. Gesteland, T. R. Cech, J. F. Atkins, Cold Spring Harbor

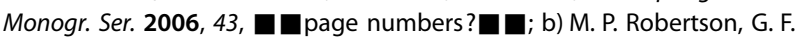
Joyce, Cold Spring Harbor Perspect. Biol. 2012, 4, a003608; c) W. Gilbert, Nature 1986, 319, 618-618. 
[3] K. Ruiz-Mirazo, C. Briones, A. de La Escosura, Chem. Rev. 2014, 114, 285 366.

[4] M. W. Powner, B. Gerland, J. D. Sutherland, Nature 2009, 459, 239-242.

[5] J. E. Hein, E. Tse, D. G. Blackmond, Nat. Chem. 2011, 3, 704-706.

[6] J. T. Sczepanski, G. F. Joyce, Nature 2014, 515, 440-442.

[7] G. F. Joyce, G. M. Visser, C. A. van Boeckel, J. H. van Boom, L. E. Orgel, J. van Westrenen, Nature 1984, 310, 602-604.

[8] a) S. Klussmann, A. Nolte, R. Bald, V. A. Erdmann, J. P. Furste, Nat. Biotechnol. 1996, 14, 1112-1115; b) D. Eulberg, S. Klussmann, ChemBiochem 2003, 4, 979-983.

[9] E. H. Ekland, J. W. Szostak, D. P. Bartel, Science 1995, 269, 364-370.

[10] F. R. Bowler, C. K. Chan, C. D. Duffy, B. Gerland, S. Islam, M. W. Powner, J. D. Sutherland, J. Xu, Nat. Chem. 2013, 5, 383-389.
[11] a) W. K. Johnston, P. J. Unrau, M. S. Lawrence, M. E. Glasner, D. P. Bartel Science 2001, 292, 1319-1325; b) A. Wochner, J. Attwater, A. Coulson, P. Holliger, Science 2011, 332, 209-212.

[12] V. B. Pinheiro, P. Holliger, Curr. Opin. Chem. Biol. 2012, 16, 245-252.

[13] a) J. A. Piccirilli, T. Krauch, S. E. Moroney, S. A. Benner, Nature 1990, 343, $33-37$; b) S. Hoshika, F. Chen, N. A. Leal, S. A. Benner, Angew. Chem. Int. Ed. 2010, 49, 5554-5557; Angew. Chem. 2010, 122, 5686-5689.

Received: January 7, 2015

Published online on $\mathbf{\square} \mathbf{\square}, 0000$ 


\section{HIGHLIGHTS}

M. Renders, V. B. Pinheiro*

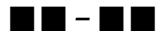

Catalysing Mirror Life

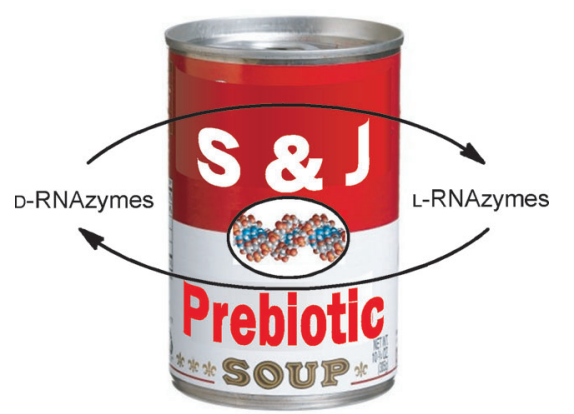

Origin of life: Although current life is homochiral (with D nucleic acids), little is known about how homochirality emerged or even if it was a necessary step. The isolation of cross-chiral nucleic acid ligases demonstrate that an early heterochiral life could have been possible. 